\title{
Analysis of the Pockels effect in ferroelectric barium titanate thin films on $\mathrm{Si}(001)$
}

\author{
Kristy J. Kormondy ${ }^{1}$, Stefan Abel $^{2}$, Florian Fallegger ${ }^{2}$, Youri Popoff ${ }^{2}$, Patrick Ponath ${ }^{1}$, Agham B. Posadas ${ }^{1}$, \\ Marilyne Sousa ${ }^{2}$, Daniele Caimi ${ }^{2}$, Heinz Siegwart ${ }^{2}$, Emanuele Uccelli $^{2}$, Lukas Czornomaz ${ }^{2}$ Chiara Marchiori $^{2}$, \\ Jean Fompeyrine ${ }^{2}$, and Alexander A. Demkov ${ }^{1}$ \\ ${ }^{1}$ Department of Physics, The University of Texas at Austin, Austin, Texas, 78712, USA \\ ${ }^{2}$ IBM Research GmbH, Zurich Laboratory, Saeumerstrasse 4, CH-8803 Rueschlikon \\ E-mail address of corresponding author: kkormond@utexas.edu
}

\section{Abstract}

High-quality epitaxial $\mathrm{BaTiO}_{3}$ (BTO) on $\mathrm{Si}$ has emerged as a highly promising material for future electro-optic (EO) devices based on BTO's large effective Pockels coefficient. We report on the EO response of BTO films deposited on $\mathrm{Si}$ by molecular beam epitaxy (MBE), and characterize the structure of these films by reflection highenergy electron diffraction and $\mathrm{x}$-ray diffraction. $\mathrm{O}_{2}$ rapid thermal anneal at $600^{\circ} \mathrm{C}$ for $30 \mathrm{~min}$ ensures full oxidation of BTO for minimal leakage current with minimal change in crystalline structure.

\section{Introduction}

The large effective Pockels coefficient $\left(r_{\text {eff }}=148\right.$ $\mathrm{pmV}^{-1}$ ) for high-quality epitaxial BTO films on $\mathrm{Si}$ distinguishes BTO as a highly promising material for integrated silicon nanophotonics [1,2]. Monolithic integration of BTO on Si is enabled by molecular beam epitaxy (MBE) through the use of buffer layers, including $\mathrm{BaO}$ [3], $\mathrm{Ba}_{0.7} \mathrm{Sr}_{0.3} \mathrm{TiO}_{3}$ [4], and most commonly, $\mathrm{SrTiO}_{3}$ (STO) $[1,5,6]$

Since the first successful integration of perovskite oxides on $\mathrm{Si}$ [7] and successive demonstration of ferroelectric functionality [6], electro-optic activity [1], and devices [2], it has become apparent that these interesting properties are strongly dependent on the crystalline structure of ferroelectric BTO. In order to achieve a strong electro-optic response, the film must have (1) the correct crystallographic orientation with respect to the applied electric field [8], and (2) low vacancy density to sustain a strong electric field [9].

In this work, we assess BTO films of thickness 40-130 nm in order to quantify the Pockels coefficient with respect to crystalline orientation. Highly insulating MBE-grown BTO films can be achieved through exposure to oxygen plasma during the deposition or post-deposition $\mathrm{O}_{2}$ rapid thermal anneal (RTA). We also examine the role of oxygen vacancies and structural defects in films of intermediate thickness $(80-90 \mathrm{~nm})$.

\section{Methods}

Epitaxial BTO films of thickness $40-130 \mathrm{~nm}$ were deposited on highly insulating (>20,000 $\Omega$ $\mathrm{cm})$ 2"-diameter Si (001) wafers through use of a $\mathrm{Sr}$ Zintl template and 4-6 nm $\mathrm{SrTiO}_{3}$ buffer layer [10], with the growth monitored by in-situ reflection high-energy electron diffraction (RHEED) (Fig. 1(a)-(c)).
We note that for the Ti-rich surface, an extra streak appears in the RHEED pattern along the $\{210\}$ direction (Fig. 1(d)), indicating a doubling of the surface unit cell size. As shown in Fig. 1(e), this extra streak disappears during the backgroundsubtracted line scan taken during one unit cell of BTO deposition $(120 \mathrm{~s})$, indicating stoichiometric BTO. For Ba-rich samples, $\mathrm{BaO}_{x}$ particles can nucleate at the surface of the as-grown film [11]. As indicated by AFM, soaking in water $[12,13]$ at room temperature for 4 hours reduces the RMS roughness from $0.763 \mathrm{~nm}$ (Fig. 2(a)) to $0.359 \mathrm{~nm}$ (Fig. 2(b)).

The backside of the Si wafer was polished for optical transmission measurements. A $300 \mathrm{~nm}$-thick tungsten layer was deposited by magnetron sputtering and subsequently dry-etched to define electrode pairs with a gap of $d=5 \mu \mathrm{m}$.

The schematic of EO measurement in transmission geometry for is illustrated in Fig. 3(a). The in-plane field modulates the refractive index of BTO, $n(E)=n_{0}-(1 / 2) r n_{0}{ }^{3} E$, which, in turn, induces a rotation $(\delta)$ of the incident linearly polarized light. To detect $\delta$, sinusoidal, alternating voltage $\left(E_{A C}=\right.$ $V_{A C} / d$ ) is applied to gain sensitivity using a lock-in amplifier. An additional DC voltage $\left(E_{\text {off }}=V_{\mathrm{DC}} / d\right)$ is also applied to align the ferroelectric domains.

\section{Results and Discussion}

Fig. 3(b) illustrates the EO response for films with varying crystalline orientation.

Since the magnitude of the response $\delta$ should scale with both the optical interaction length and the magnitude of the applied AC field, we compare the field- and thickness-normalized optical rotation $\delta^{\prime}=\delta /\left(E_{A C} \cdot t\right)$, where $t$ is the thickness of the BTO layer. The thickness of the STO and Si layers are not included, as no Pockels effect is expected for materials with inversion symmetry [14].

The strongest optical response $\delta$ ' is observed when the BTO tetragonal axis is oriented in-plane (" $a$-axis", solid squares). The hysteresis of $\delta$ ' vs DC offset electric field $E_{\text {off }}$ is also characteristic of reorientation of multiple in-plane ferroelectric domains [1]. Alternatively, a vanishing EO response is measured when the BTO tetragonal axis is oriented out-of-plane ("c-axis", solid circles). Finally, a strong response is observed even for the "mixed" $a$ - and $c$-axis film (open triangles), attributable to the fraction of $a$-axis domains.

We also identify post-deposition $\mathrm{O}_{2}$ rapid thermal anneal (RTA) as a route to increase the 
fraction of $a$-axis BTO. Fig. 4 illustrates the structural changes of an as-grown $c$-axis film after successive rounds of RTA $\left(500-850^{\circ} \mathrm{C}, 50^{\circ} \mathrm{C}\right.$ increments, $30 \mathrm{~min}$ each). A reduced FWHM of the (002) BTO Bragg peak rocking curve indicates improved crystallinity. Between the $700^{\circ} \mathrm{C}$ and $850^{\circ} \mathrm{C}$ annealing steps, the out-of-plane lattice constant decreases from $4.03 \AA$ to $3.99 \AA$, indicating a transition from $c$ - to $a$-axis orientation.

An additional role of $\mathrm{O}_{2}$ RTA is the healing of oxygen vacancies, electrically active defects which can impede build-up of a strong electric field. To illustrate, we compare (Fig. 5) two $90 \mathrm{~nm}$ BTO/STO/Si samples, one deposited in molecular oxygen and the other under oxygen plasma-assisted conditions. The sample as-grown in molecular $\mathrm{O}_{2}$ exhibited a high leakage (Fig. 5, grey squares). While air anneal up to $300^{\circ} \mathrm{C}$ and RTA at $400^{\circ} \mathrm{C}$ resulted in minimal change, RTA at $600^{\circ} \mathrm{C}$ for 30 min reduced leakage current by 4 orders of magnitude, ensuring a strong electric field and enabling EO measurement (Fig. 5, black squares). A similarly low leakage current is measured for two as-grown oxygen plasma-assisted samples, one with Ti evaporated from a Knudsen cell (K-cell) at a rate of $4 \AA / \mathrm{min}$ (Fig. 5, red circles) while the other used an electron-beam evaporated (e-beam) Ti source at a rate of $12 \AA / \mathrm{min}$ (Fig. 5, blue triangles).

From EO measurements of the three highly insulating samples, we calculate an effective Pockels coefficient, $r_{\text {eff }}=\left(2 \delta_{\max }\right) \lambda\left(\pi n^{3} t E_{\mathrm{AC}}\right)$, where $\delta_{\max }$ is the maximum induced rotation angle in radians, $\lambda=1550 \mathrm{~nm}$ is the wavelength of incident light, $n=2.26$ is the refractive index of the film as determined by spectroscopic ellipsometry, $t$ the total thickness of the BTO layer [15]. Results are summarized in Table 1 for comparison with previous works, for example $\sim 2 \mathrm{pm} / \mathrm{V}$ for strained $\mathrm{Si}, \sim 30 \mathrm{pm} / \mathrm{V}$ for $\mathrm{LiNbO}_{3}$ on $\mathrm{Si}$, and $148 \mathrm{pm} / \mathrm{V}$ for BTO/Si [1,16,17]. By comparing the effective Pockels coefficient for the mixed films, we see that the strongest response is observed for the e-beam $\mathrm{Ti}$ sample. XRD of this sample indicates a large fraction of in-plane oriented BTO as compared to the two K-cell Ti samples (Fig. 5(b)).

\section{Conclusion}

In conclusion, for epitaxial $\mathrm{BTO} / \mathrm{Si}$, we measure an increasing EO response for films with increasing $a$ axis fraction and low leakage current. We further identify post-deposition $\mathrm{O}_{2}$ RTA as beneficial to achieve (1) in-plane tetragonal axis orientation and (2) full oxidation of BTO. Future work should focus on numerical simulations (based on the Pockels tensor, domain structure, field orientation, and angle of incidence) for modeling the response of $c$-axis domains. Such analysis might allow more accurate modeling of the electro-optic response of $c$ - and $a$-axis domains in films with mixed domains to extract the full Pockels tensor of these films.

\section{Acknowledgements}

This work was supported by the National Science Foundation (IRES- 1358111), the European Commission (FP7-ICT-2013-11-619456-SITOGA) and the Air Force Office of Scientific Research (FA9550-12-10494).

\section{References}

[1] S. Abel, T. Stöferle, C. Marchiori, C. Rossel, M.D. Rossell, R. Erni, D. Caimi, M. Sousa, A. Chelnokov, B.J. Offrein, J. Fompeyrine, Nat. Commun. 4 (2013) 1671.

[2] C. Xiong, W.H.P. Pernice, J.H. Ngai, J.W. Reiner, D. Kumah, F.J. Walker, C.H. Ahn, H.X. Tang, Nano Lett. 14 (2014) 1419.

[3] R.A. McKee, F.J. Walker, M.F. Chisholm, Science 293 (2001) 468.

[4] V. Vaithyanathan, J. Lettieri, W. Tian, A. Sharan, A. Vasudevarao, Y.L. Li, A. Kochhar, H. Ma, J. Levy, P. Zschack, J.C. Woicik, L.Q. Chen, V. Gopalan, D.G. Schlom, J. Appl. Phys. 100 (2006) 024108.

[5] F. Niu, B.W. Wessels, J. Vac. Sci. Technol. B Microelectron. Nanom. Struct. 25 (2007) 1053

[6] C. Dubourdieu, J. Bruley, T.M. Arruda, A. Posadas, J. Jordan-Sweet, M.M. Frank, E. Cartier, D.J. Frank, S. V Kalinin, A.A. Demkov, V. Narayanan, Nat. Nanotechnol. 8 (2013) 748.

[7] R.A. McKee, F.J. Walker, J.R. Conner, E.D. Specht, D.E. Zelmon, Appl. Phys. Lett. 59 (1991) 782.

[8] B.W. Wessels, Annu. Rev. Mater. Res. 37 (2007) 659.

[9] R. Pan, Y. He, M. Li, P. Li, P. Liu, Z. Xia, Mater. Sci. Eng. B 188 (2014) 84.

[10] R.A. McKee, F.J. Walker, M.F. Chisholm, Phys. Rev. Lett. 81 (1998) 3014.

[11] A. Barbier, C. Mocuta, D. Stanescu, P. Jegou, N Jedrecy, H. Magnan, J. Appl. Phys. 112 (2012) 114116.

[12] R.C. Hatch, K.D. Fredrickson, M. Choi, C. Lin, H. Seo, A.B. Posadas, A.A. Demkov, J. Appl. Phys. 114 (2013) 103710.

[13] C. Raisch, T. Chassé, C. Langheinrich, a. Chassé, J. Appl. Phys. 112 (2012) 073505.

[14] G.T. Reed, C.E. Jason Png, Mater. Today 8 (2005) 40

[15] D.H. Reitze, E. Haton, R. Ramesh, S. Etemad, D.E. Leaird, T. Sands, Z. Karim, A.R. Tanguay, Appl. Phys. Lett. 63 (1993) 596.

[16] C. Schriever, C. Bohley, J. Schilling, R.B. Wehrspohn, Materials, 5 (2012) 889.

[17] P. Rabiei, P. Gunter, Appl. Phys. Lett. 85 (2004) 4603. 

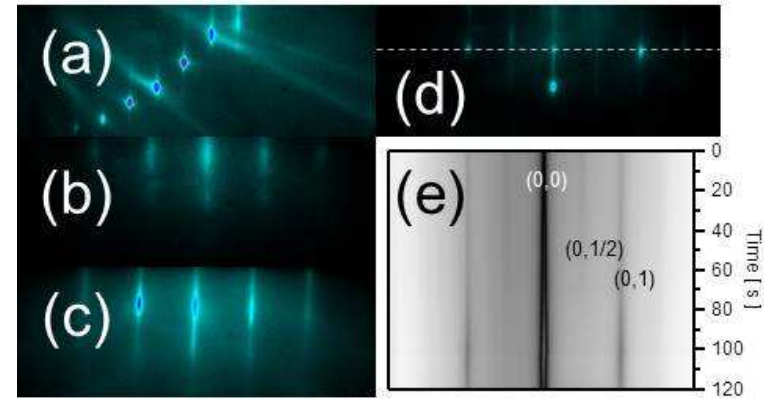

Fig. 1: RHEED patterns for (a) Sr Zintl template on Si along Si \{110\}, (b) $6 \mathrm{~nm}$ STO along STO \{110\}, and (c) $90 \mathrm{~nm}$ BTO along \{110\}. (d) The BTO <210> surface unit cell was monitored during growth. An extra streak appears in the RHEED pattern for the Ti-rich surface, indicating a doubling of the surface unit cell size. (e) In a line scan taken during one unit cell of BTO deposition (120 s), the extra streak disappears for stoichiometric BTO.

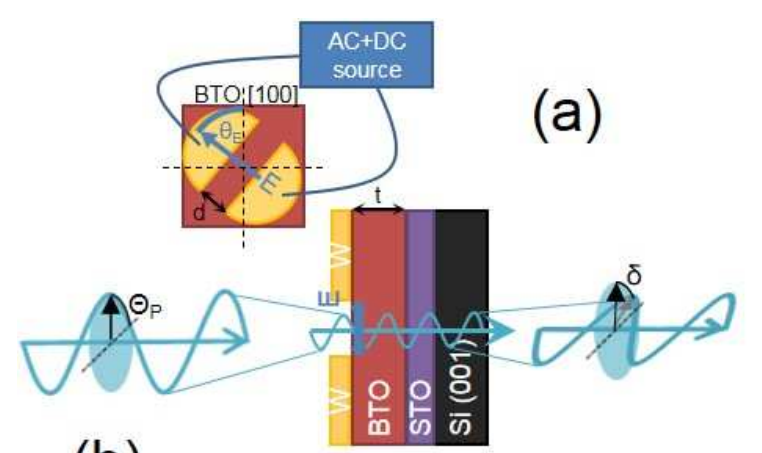

(b)

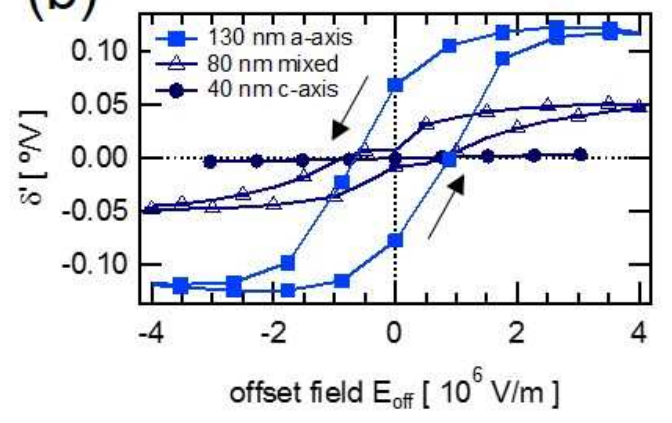

Fig. 3: (a) Electro-optical characterization quantifies rotation $(\delta)$ of the polarization $\left(\vartheta_{p}\right)$ of a laser beam transmitted through pairs of lithographically defined electrodes. (b) Thickness-normalized electro-optic response $\delta=\delta /\left(E_{A C} \cdot t\right)$. as a function of offset (DC) field for a-axis, mixed, and c-axis films.

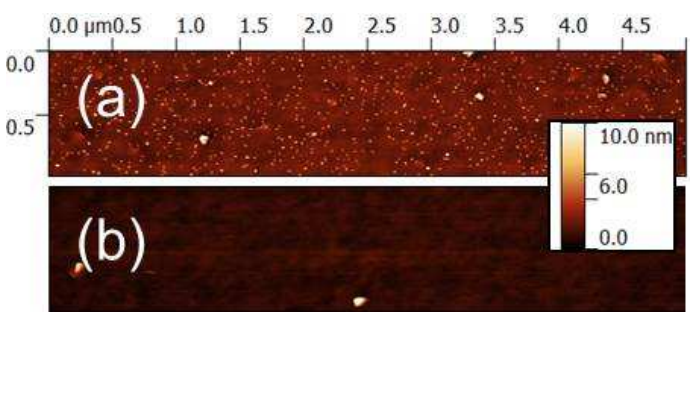

Fig. 2: Atomic force microscopy (AFM) of the as-grown sample (a) reveals many small particles (presumably $\mathrm{BaOx}$ ) on the surface. After soaking in water for 4 hours (b), few particles remain.
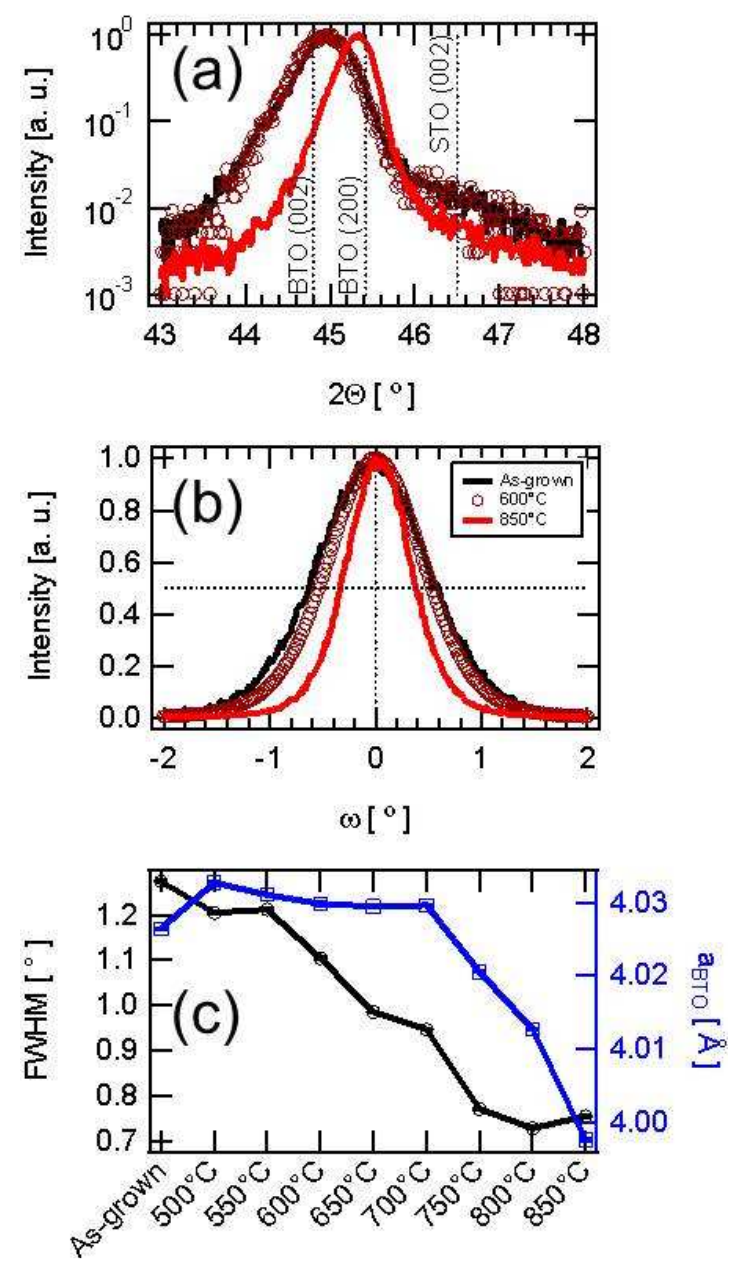

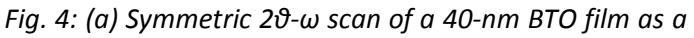
sample undergoes a series of $30 \mathrm{~min}$ RTA in 02 at incremental temperatures $\left(500-850^{\circ} \mathrm{C}\right)$. (b) Corresponding rocking curves about the (002) BTO Bragg peak. (c) Corresponding FWHM and out-of-plane lattice constants over the annealing series 

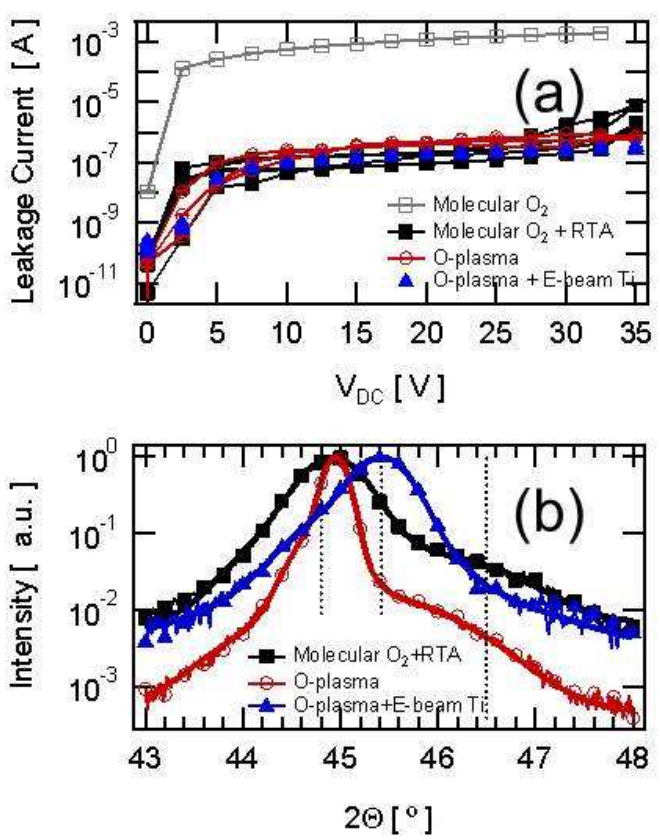

Fig. 5: (a) Leakage current and (b) symmetric $2 \vartheta-\omega$ scan for a series of films with varied growth conditions.

\begin{tabular}{cccccccc}
\hline \hline Sample & $\begin{array}{c}\text { Oxygen } \\
\text { source }\end{array}$ & $\begin{array}{c}\mathrm{Ti} \\
\text { source }\end{array}$ & $\begin{array}{c}\text { Thickness } \\
(\mathrm{nm})\end{array}$ & $\begin{array}{c}\text { Leakage current } \\
(\mathrm{A})\end{array}$ & $\begin{array}{c}r_{\text {eff }} \\
(\mathrm{pm} / \mathrm{V})\end{array}$ & FWHM $\left(^{\circ}\right)$ & $a_{\mathrm{BTO}}(\mathrm{A})$ \\
\hline $\mathrm{A}$ & Molecular & K-cell & 90 & $10^{-3}$ & $\mathrm{~N} / \mathrm{A}$ & $0.75 \pm 0.07$ & $4.023 \pm 0.008$ \\
$\mathrm{~A}^{\prime}$ & Anneal & K-cell & 90 & $10^{-7}$ & 46 & $0.79 \pm 0.11$ & $4.031 \pm 0.009$ \\
$\mathrm{~B}$ & Plasma & K-cell & 90 & $10^{-7}$ & 39 & $0.40 \pm 0.05$ & $4.028 \pm 0.004$ \\
$\mathrm{C}$ & Plasma & E-gun & 80 & $10^{-7}$ & 50 & $\mathrm{~N} / \mathrm{A}$ & $3.978 \pm 0.009$ \\
\hline \hline
\end{tabular}

Table 1: Pockels coefficients for samples grown under various growth conditions. 


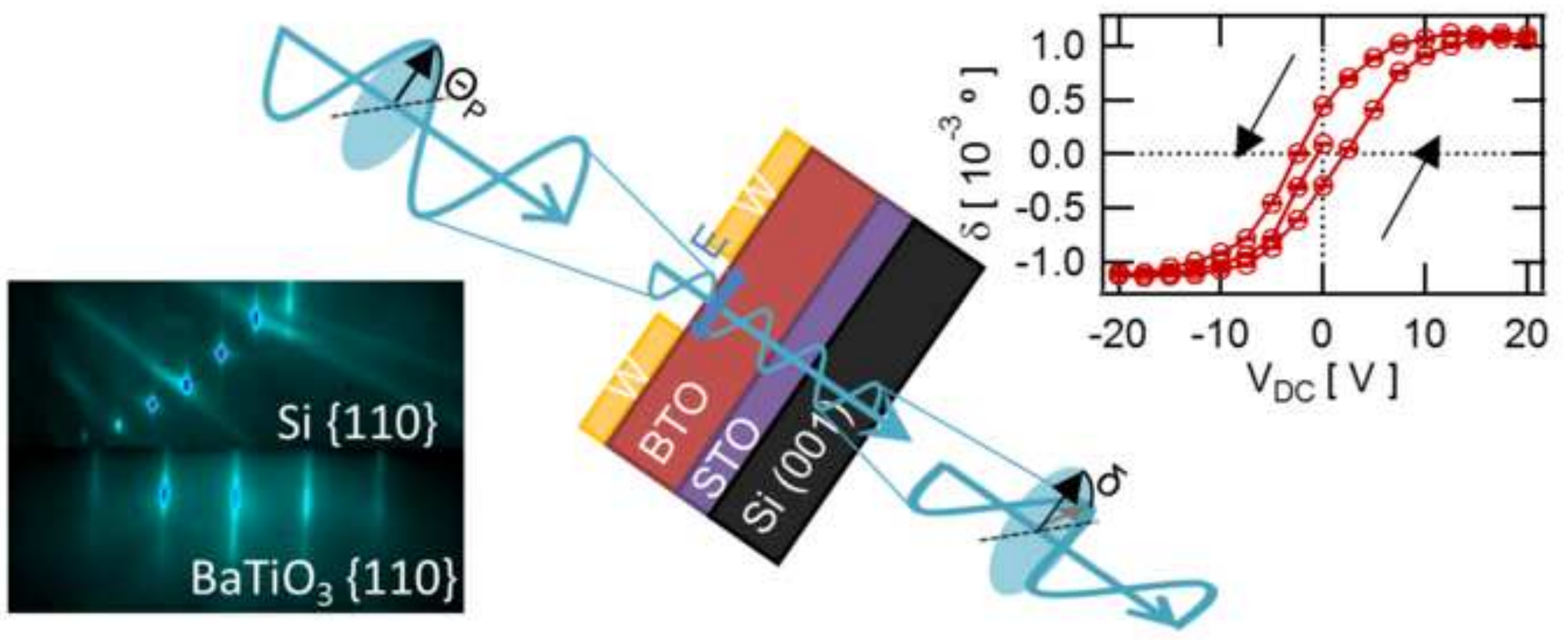

